\title{
Antibodies against specific extractable nuclear antigens (ENAs) as diagnostic and prognostic tools and inducers of a profibrotic phenotype in cultured human skin fibroblasts: are they functional?
}

\author{
Claudio Corallo ${ }^{1 *}$, Sara Cheleschi ${ }^{2}$, Maurizio Cutolo ${ }^{3}$, Stefano Soldano ${ }^{3}$, Antonella Fioravanti $^{2}$, Nila Volpi ${ }^{2}$,
} Daniela Franci ${ }^{1}$, Ranuccio Nuti ${ }^{1}$ and Nicola Giordano ${ }^{1}$

\begin{abstract}
Background: The importance of systemic sclerosis (SSC) autoantibodies for diagnosis has become recognized by their incorporation into the 2013 ACR/EULAR classification criteria. Clear prognostic and phenotypic associations with cutaneous subtype and internal organ involvement have been also described. However, little is known about the potential of autoantibodies to exert a direct pathogenic role in SSc. The aim of the study is to assess the pathogenic capacity of anti-DNA-topoisomerase I (anti-Topo-I) and anti-centromeric protein B (anti-Cenp-B) autoantibodies to induce pro-fibrotic markers in dermal fibroblasts.

Methods: Dermal fibroblasts were isolated from unaffected and affected skin samples of $(n=10)$ limited cutaneous SSC (LCSSC) patients, from affected skin samples of diffuse cutaneous (DCSSC) patients $(n=10)$ and from healthy subjects $(n=20)$. Fibroblasts were stimulated with anti-Topo-l, anti-Cenp-B lgGs, and control lgGs in ratios 1:100 and 1:200 for $24 \mathrm{~h}$. Cells were also incubated with $10 \%$ SSC anti-Topo- ${ }^{+}$and anti-Cenp- $\mathrm{B}^{+}$whole serum and with $10 \%$ control serum for $24 \mathrm{~h}$. Viability was assessed by MTT test, while apoptosis was assessed by flow cytometry. Activation of pro-fibrotic genes ACTA2, COL1A1, and TAGLN was evaluated by quantitative real-time PCR (qPCR), while the respective protein levels alpha-smooth-muscle actin (a-SMA), type-l-collagen (Col-l), and transgelin (SM22) were assessed by immunocytochemistry (ICC).

Results: MTT showed that anti-Cenp-B/anti-Topo-I IgGs and anti-Cenp-B ${ }^{+}$/anti-Topo-I ${ }^{+}$sera reduced viability (in a dilutiondependent manner for $\operatorname{lgGs}$ ) for all the fibroblast populations. Apoptosis is induced in unaffected LCSSc and control fibroblasts, while affected LCSSC/DcSSc fibroblasts showed apoptosis resistance. Basal mRNA (ACTA2, COL1A1, and TAGLN) and protein (a-SMA, Col-1, and SM22) levels were higher in affected LCSSC/DCSSc fibroblasts compared to LCSSC unaffected and to control ones. Stimulation with anti-Cenp-B/anti-Topo-l lgGs and with anti-Cenp-B $/$ anti-Topo-l ${ }^{+}$sera showed a better induction in unaffected LCSSC and control fibroblasts. However, a statistically significant increase of all pro-fibrotic markers is reported also in affected LCSSC/DCSSc fibroblasts upon stimulation with both lgGs and sera.
\end{abstract}

Conclusions: This study suggests a pathogenic role of SSc-specific autoantibodies to directly induce pro-fibrotic activation in human dermal fibroblasts. Therefore, besides the diagnostic and prognostic use of those autoantibodies, these data might further justify the importance of immunosuppressive drugs in the early stages of the autoimmune disease, including SSc.

Keywords: Systemic sclerosis, Fibrosis, Autoantibodies, Fibroblasts, Centromeric protein B, Topoisomerase I

\footnotetext{
* Correspondence: corallo.claudio@gmail.com

'Scleroderma Unit, Department of Medicine, Surgery and Neurosciences,

University of Siena, Siena, Italy

Full list of author information is available at the end of the article
}

(c) The Author(s). 2019 Open Access This article is distributed under the terms of the Creative Commons Attribution 4.0 International License (http://creativecommons.org/licenses/by/4.0/), which permits unrestricted use, distribution, and reproduction in any medium, provided you give appropriate credit to the original author(s) and the source, provide a link to the Creative Commons license, and indicate if changes were made. The Creative Commons Public Domain Dedication waiver (http://creativecommons.org/publicdomain/zero/1.0/) applies to the data made available in this article, unless otherwise stated. 


\section{Background}

Systemic sclerosis (scleroderma, SSc) is a rare and heterogeneous autoimmune disease characterized by progressive fibrosis of the skin and internal organs such as lungs, heart, kidneys, and gastrointestinal tract, coupled to widespread vascular alterations [1]. The main abnormalities of SSc are related to the connective tissue, in which the excessive production of collagen and other extracellular matrix components are responsible for a progressive and, so far irreversible, fibrosis [2]. The clinical phenotype of SSc varies between two main distinct subsets according to the extent of the skin involvement [3]: limited cutaneous systemic sclerosis (LcSSc) in which skin thickening is mainly restricted to the face, fingers, and forearms [4] and diffuse cutaneous systemic sclerosis (DcSSc) in which skin lesions are observed on the trunk and over the elbow and/or knee [5]. It is well known that SSc has an autoimmune etiology: antinuclear antibodies (ANAs) are detected in more than 95\% of patients [6] and the presence of several potentially pathogenic auto-antibodies targeting various extractable nuclear antigens (ENAs) or other auto-antigens is also reported [7]. ANAs in SSc are divided into two categories: SSc-specific ANAs and SSc-associated ANAs [8]. SSc-specific ANAs are detected in SSc patients and rarely found in other connective tissue diseases or in healthy subjects [9]. They include anti-centromere (ACAs) and anti-DNA topoisomerase I (Topo-I) antibodies mainly, but also anti-RNA polymerase III (RNAP), anti-U3 ribonucleoprotein (RNP), anti-Th/To, anti-U11/U12 RNP, anti-eukaryotic initiation factor 2B (eIF2B), anti-U1 RNP, anti-PM-Scl, anti-Ku, and antiRuvBL1/2 antibodies for the minor component. On the other hand, SSc-associated ANAs are not specific to SSc but they can occasionally coexist with other connective tissue disease-related antibodies [10, 11]. The two main subsets of SSc (LcSSc and DcSSc) do not reflect only a clinical classification [3], but they are usually associated with a precise autoimmune pattern: in fact, ACAs and in particular anti-centromere B (anti-Cenp-B) antibodies are predominantly associated with LcSSc, while antiTopo-I with DcSSc [12]. In fact, while the other SScspecific or SSc-associated antibodies can be found in both LcSSc and DcSSc, it is less frequent to find LcSSc patients with anti-Topo-I antibodies and DcSSc patients with anti-Cenp-B antibodies [13]. The utility of SScspecific antibodies for both diagnostic and prognostic purposes has been fully elucidated [14]. In fact, the ACR/EULAR 2013 classification criteria now include the presence of ACAs, anti-Topo-I, and anti-RNAP-III antibodies as one of the items to overcome the disadvantages of the 1980 ACR preliminary classification criteria $[15,16]$. Regarding the prognostic utility, the faster progression of the disease in SSc-specific antibody-positive patients versus SSc-specific antibody-negative ones has been demonstrated [17]. ANA-negative SSc patients (5\%) represent one unique subgroup mainly characterized by male subjects with less vasculopathy, such as digital ulcers and pulmonary arterial hypertension (PAH), and with lower gastrointestinal involvement [18]. Whether ANA-negative SSc patients have other nonnuclear circulating antibodies has not been currently elucidated [19]. Taken into account the abovementioned diagnostic and prognostic utilities of SSc-specific antibodies, the goal of the present study is to investigate whether those antibodies could have a direct pathogenic effect on in vitro cultured human fibroblasts.

\section{Materials and methods}

\section{Patients and cell cultures}

Ten patients with LcSSc and ten patients with DcSSc who fulfilled the 2013 ACR/EULAR classification criteria for SSc $[15,16]$, and ten age- and sex-matched voluntary healthy subjects were recruited from the Internal Medicine and Rheumatology Units of the University Hospital of Siena in accordance with the Declaration of Helsinki, after obtaining signed informed consent and following the local Ethical Board Committee approval. Clinical and demographical characteristics of SSc patients are reported in Table 1. Blood was collected from SSc patients and from healthy subjects, and serum samples were checked for the presence of autoantibodies according to normal diagnostic procedures. Skin biopsies were performed using a 3-mm punch on the affected midforearm of patients with LcSSc/DcSSc. Unaffected areas of skin from the same LcSSc patients and control skin (site-matched) from gender- and age-matched healthy subjects were also evaluated. The LcSSc patients' unaffected skin was defined by clinical palpation and graded as zero on the modified Rodnan skin score [20]. Fibroblasts were isolated from skin specimens by enzymatic digestion. Briefly, explants were de-epidermized using a dispase solution (dispase activity $14 \mathrm{U} / \mathrm{mL}$ ) (Sigma-Aldrich, St. Louis, MO, USA) for $2 \mathrm{~h}$ at $37^{\circ} \mathrm{C}$ and then were dissolved into a type IV collagenase solution $(2.4 \mathrm{U} / \mathrm{mL})$ (Sigma-Aldrich) for $3 \mathrm{~h}$.

The obtained cell suspension was filtered twice using $70-\mu \mathrm{m}$ nylon meshes, washed, and centrifuged for $5 \mathrm{~min}$ at $700 \times g$. The viability was assessed by Trypan Blue (Sigma-Aldrich) test identifying 90 to $95 \%$ cell survival. Fibroblasts were recovered, seeded into $10-\mathrm{cm}$ diameter tissue culture plates, and were expanded twice and cultured in a monolayer incubator with $5 \% \mathrm{CO}_{2}$ and $90 \%$ humidified atmosphere at $37^{\circ} \mathrm{C}$ until confluence. Cells were grown in Dulbecco's modified Eagle medium (DMEM) (Euroclone, Milan, Italy), containing 10\% fetal bovine serum (FBS) (Euroclone), with $200 \mathrm{U} / \mathrm{mL}$ penicillin and $200 \mu \mathrm{g} / \mathrm{mL}$ streptomycin (Sigma-Aldrich) and 2 
Table 1 Demographic and clinical characteristics of patients at the time of biopsy collection

\begin{tabular}{|c|c|c|c|c|c|c|c|c|}
\hline Patients & Subset & Age (years) & Gender (M/F) & $\begin{array}{l}\text { Disease duration } \\
\text { (years) }\end{array}$ & Autoantibody & mRSS & Organ involvement & Therapy \\
\hline 1 & LCSSC & 57 & $F$ & 12 & Anti-Cenp-B & 17 & Lung (ILD), digital ulcers & ERAs, prednisone \\
\hline 2 & LCSSC & 62 & $F$ & 5 & Anti-Cenp-B & 4 & Esophagus & $\mathrm{Ca} 2+$ antagonists, PPIs \\
\hline 3 & LCSSC & 70 & M & 9 & Anti-Cenp-B & 14 & Lung (ILD), esophagus & ERAs, PPIs, MMF \\
\hline 4 & LCSSC & 67 & $\mathrm{~F}$ & 10 & Anti-Cenp-B & 5 & Esophagus & $\mathrm{Ca}_{2+}$ antagonists, PPIs \\
\hline 5 & LCSSC & 48 & $\mathrm{~F}$ & 8 & Anti-Cenp-B & 14 & Lung (ILD) & ERAs, Prednisone, MMF \\
\hline 6 & LCSSC & 55 & $F$ & 11 & Anti-Cenp-B & 7 & Lung (ILD) & ERAs, Prednisone \\
\hline 7 & LCSSC & 58 & $\mathrm{~F}$ & 13 & Anti-Cenp-B & 17 & Lung (ILD), esophagus & ERAs, PPIs, MMF \\
\hline 8 & LCSSC & 44 & $\mathrm{~F}$ & 2 & Anti-Cenp-B & 7 & Lung (PAH), esophagus & $\mathrm{Ca}_{2+}$ antagonists, PPIs, prostanoids \\
\hline 9 & LCSSC & 66 & $\mathrm{~F}$ & 7 & Anti-Cenp-B & 14 & $\begin{array}{l}\text { Digital ulcers } \\
\text { esophagus }\end{array}$ & ERAs, PPIs \\
\hline 10 & LCSSC & 60 & $\mathrm{~F}$ & 9 & Anti-Cenp-B & 5 & Esophagus & $\mathrm{Ca}_{2+}$ antagonists, PPIs \\
\hline 11 & DcSSc & 51 & $\mathrm{~F}$ & 12 & Anti-Topo-I & 7 & Lung (PAH), digital ulcers & $\mathrm{Ca}_{2+}$ antagonists, ERAs, prostanoids \\
\hline 12 & DcSSC & 72 & M & 11 & Anti-Topo-I & 17 & Lung (PAH), digital ulcers & ERAs, prostanoids, MMF \\
\hline 13 & DcSSc & 64 & $\mathrm{~F}$ & 7 & Anti-Topo-I & 17 & Lung $(\mathrm{PAH})$, digital ulcers & ERAs, prostanoids, MMF \\
\hline 14 & DCSSC & 63 & M & 13 & Anti-Topo-I & 12 & Lung (ILD) & ERAs, prednisone, MMF \\
\hline 15 & DcSSc & 50 & $\mathrm{~F}$ & 6 & Anti-Topo-I & 9 & Lung (ILD), esophagus & $\mathrm{Ca}_{2+}$ antagonists, PPIs \\
\hline 16 & DcSSc & 68 & $F$ & 15 & Anti-Topo-I & 14 & Lung (ILD) & ERAs, prednisone, MMF \\
\hline 17 & DcSSc & 59 & $F$ & 14 & Anti-Topo-I & 17 & Esophagus & $\mathrm{Ca}_{2+}$ antagonists, PPIs \\
\hline 18 & DcSSc & 69 & M & 7 & Anti-Topo-I & 17 & Lung (PAH) & ERAs, prostanoids \\
\hline 19 & DCSSC & 49 & $\mathrm{~F}$ & 9 & Anti-Topo-I & 14 & Lung (PAH), esophagus & ERAs, prostanoids, PPIs \\
\hline 20 & DcSSc & 61 & $\mathrm{~F}$ & 11 & Anti-Topo-I & 17 & Lung (ILD), esophagus & $\mathrm{Ca}_{2+}$ antagonists, PPIs, MMF \\
\hline
\end{tabular}

Abbreviations: Cenp-B centromeric protein B, ERAs endothelin receptor antagonists, ILD interstitial lung disease, MMF mycophenolate mofetil, $m R S S$ modified Rodnan skin score, PPIs proton pump inhibitors, $P A H$ pulmonary arterial hypertension, Topo-I topoisomerase I

mM glutamine (Sigma-Aldrich). The medium was changed every 3-4 days. The fibroblast morphology was examined daily with an inverted microscope (Olympus IMT-2, Tokyo, Japan) to guarantee their phenotypic stability preserved. For each single experiment, a cell culture from a unique donor was used.

\section{Fibroblast treatment}

Fibroblasts at the third passage were employed for the experiments. Twelve hours before the experiments, cells were harvested in a serum-free medium and cultured in $75 \mathrm{~cm}^{2}$ flasks (Euroclone, Milan, Italy).

Human polyclonal anti-centromere B (anti-Cenp-B) and anti-DNA topoisomerase I (anti-Topo-I) (Abcam, Cambridge, UK) were first dissolved in phosphatebuffered saline (PBS) (Euroclone), according to the manufacturer's instructions, and then they were diluted in the culture medium immediately before the treatment to reach the final dilution required $(1: 100$ and 1:200). The cells were treated with the conditioned media containing the selected dilutions of antiCenp-B and anti-Topo I and 10\% SSc anti-Topo- $\mathrm{I}^{+}$, anti-Cenp- $\mathrm{B}^{+}$serum for $24 \mathrm{~h}$. The final concentrations were chosen based on the best results obtained in terms of viability (data not shown). To evaluate the effect of antibodies directed against nuclear proteins which are not involved in SSc pathophysiology, a human anti-histone $\mathrm{H} 3$ antibody (Abcam) was also tested in parallel in control fibroblasts only (Additional file 1).

After the treatment, the media were removed, centrifuged, and stored at $-80{ }^{\circ} \mathrm{C}$; the fibroblasts were immediately processed to carry out cell viability assay, flow cytometry analysis, quantitative real-time PCR, and immunofluorescence analysis.

\section{MTT assay}

The viability of the cells was evaluated immediately after the treatment by MTT assay. Fibroblasts from LcSSc/ DcSSc patients and healthy subjects were seeded in 12well plates $\left(8 \times 10^{4}\right.$ cells/well $)$ for $24 \mathrm{~h}$ in DMEM with $10 \%$ FBS. Then, the medium was removed, and the cells were cultured in DMEM with $0.5 \%$ FBS usually used during the treatment procedure. After that, the cells were incubated for $3 \mathrm{~h}$ at $37^{\circ} \mathrm{C}$ in a culture medium containing $10 \%$ of $5 \mathrm{mg} / \mathrm{mL}$ MTT (3-[4,4-dimethylthiazol-2-yl]-2,5-diphenyl-tetrazoliumbromide) (SigmaAldrich). After the period of incubation, the medium was removed and $0.2 \mathrm{~mL}$ of dimethyl sulfoxide (DMSO) 
(Rottapharm Biotech, Monza, Italy) was added to each well to solubilize the formazan crystals. The absorbance was measured at $570 \mathrm{~nm}$ in a microplate reader (BioTek Instruments, Inc., Winooski, VT, USA). A control well without cells was employed for blank measurement. The percentage of survival cells was evaluated as follows: \% of survival cells $=$ (absorbance of considered sample) / (absorbance of control) $\times 100$. The experiments were performed on sub-confluent cell cultures in order to prevent contact inhibition which can condition the results. Data were normalized and reported as optical density (OD) units per $10^{4}$ adherent cells.

\section{Detection of apoptosis}

The evaluation of apoptotic cells was developed by using Annexin V-FITC and propidium iodide (PI) (Thermo Fisher Scientific, Milan, Italy). Fibroblasts from LcSSc/DcSSc patients and healthy subjects were seeded in 12-well plates $\left(8 \times 10^{4}\right.$ cells/well $)$ for $24 \mathrm{~h}$ in DMEM with $10 \%$ FBS. Then, the medium was removed, and the cells were cultured in DMEM with $0.5 \%$ FBS usually used during the treatment procedure described before. After that, the fibroblasts were washed and harvested by using trypsin, collected into cytometry tubes, and centrifuged at $1500 \mathrm{rpm}$ for 10 min. The supernatant was replaced, and the pellet was resuspended in $100 \mu \mathrm{L}$ of $1 \times$ Annexin-binding buffer, $5 \mu \mathrm{L}$ of Alexa Fluor 488 annexin-V conjugated to fluorescein (green fluorescence), and $1 \mu \mathrm{L}$ of $100 \mu \mathrm{g} / \mathrm{mL}$ PI working solution. Cells were incubated at room temperature for $15 \mathrm{~min}$ in the dark. Then, $600 \mu \mathrm{L}$ of $1 \times$ Annexin-binding buffer was added before the analysis at flow cytometer. A total of 10,000 events $\left(1 \times 10^{4}\right.$ cells per assay) were measured by the instrument. The obtained results were analyzed with Cell Quest software (Version 4.0, Becton Dickinson, San Jose, CA, USA). The evaluation of apoptosis was carried out considering staining cells simultaneously with Alexa Fluor 488 annexin-V and PI; this allowed to discriminate intact cells (annexin-V and PInegative), early apoptosis (annexin-V-positive and PInegative), and late apoptosis (annexin-V and PI positives) [21]. The results were normalized per $10^{4}$ cells and expressed as a ratio of positive cells to each dye (total apoptosis), and the data were represented as the mean of three independent experiments (mean \pm standard deviation (SD)). To determine the impact of apoptosis on fibroblast pro-fibrotic activation, inhibitor of apoptosis (IAP) AZD 5582 dihydrochloride (Sigma-Aldrich) compound was added to control fibroblasts $2 \mathrm{~h}$ before the stimulation with anti-Cenp-B (1:100) and anti-Topo-I (1:100) IgGs. The final concentration chosen is $50 \mathrm{nM}$ after appropriate dose finding (Additional file 1).

\section{RNA isolation and quantitative real-time PCR}

Fibroblasts from LcSSc/DcSSc patients and healthy subjects were seeded in 6-well dishes at a starting density of $6 \times 10^{6}$ cells/well for $24 \mathrm{~h}$ in DMEM with $10 \%$ FBS. Then, the medium was removed, and the cells were cultured in DMEM with 0.5\% FBS usually used during the treatment procedure.

Total RNA was extracted using TriPure Isolation Reagent (Euroclone) according to the manufacturer's instructions and was stored at $-80^{\circ} \mathrm{C}$. The concentration, purity, and integrity of RNA were evaluated by measuring the OD at $260 \mathrm{~nm}$ and the $260 / 280$ and $260 / 230$ ratios by Nanodrop-1000 (Celbio, Milan, Italy). The quality of RNA was verified by electrophoresis on agarose gel (Flash Gel System, Lonza, Rockland, ME, USA). Reverse transcription for target genes was carried by QuantiTect Reverse Transcription Kit (Qiagen, Hilden, Germany), according to the manufacturer's instructions.

Then, target genes were examined by real-time PCR by using QuantiFast SYBR Green PCR (Qiagen) kit. A list of the used primers is reported in Table 2. All qPCR reactions were achieved in glass capillaries by a LightCycler 1.0 (Roche Molecular Biochemicals, Mannheim, Germany) with LightCycler Software Version 3.5. The reaction procedure for target gene amplification was performed at 5 in at $95^{\circ} \mathrm{C}, 40$ cycles of $15 \mathrm{~s}$ at $95^{\circ} \mathrm{C}$, and $30 \mathrm{~s}$ at $60^{\circ} \mathrm{C}$. In the final step of the protocol, the temperature was raised from 60 to $95^{\circ} \mathrm{C}$ at $0.1{ }^{\circ} \mathrm{C} /$ step to plot the melting curve.

To further analyze the dissociation curves, we visualized the amplicon lengths in an agarose gel to confirm the correct amplification of the resulting PCR products. For the data analysis, the $\mathrm{Ct}$ values of each sample and the efficiency of the primer set were calculated through LinReg Software [22] and then converted into relative quantities and normalized using the Pfaffl model [23]. The normalization was performed considering human glyceraldehyde 3-phosphate dehydrogenase (GAPDH) as the housekeeping gene. This gene was chosen according to geNorm software version 3.5 [24].

\section{Immunofluorescence}

Fibroblasts derived from LcSSc/DcSSc patients and from healthy subjects were plated in coverslips in Petri dishes $(35 \times 10 \mathrm{~mm})$ at a starting low density of $4 \times 10^{4}$ cells/chamber, to prevent possible cell overlapping, and re-suspended in $2 \mathrm{~mL}$ of culture medium until $80 \%$ of confluence. The cells were processed after 24 $\mathrm{h}$ of treatment to evaluate the cytoplasmic localization of $\alpha$-SMA, Col-I, and SM22. The fibroblasts were washed in PBS (Euroclone) and then fixed in 4\% paraformaldehyde solution (Sigma-Aldrich) for $15 \mathrm{~min}$ at room temperature. Afterwards, to permeabilize cell membranes, cells were incubated in Triton-X $1000.2 \%$ solution for $30 \mathrm{~min}$ at room 
Table 2 Primers used for RT-qPCR

\begin{tabular}{lccc}
\hline & Cat. no. (Qiagen) & Forward sequence & Reverse sequence \\
\hline Target gene & & & CAGATCCAGACGCATGATGGCA \\
ACTA2 & QT00088102 & CTATGCCTCTGGACGCACAACT & AGCCTCTCCATCTITGCCAGCA \\
COL1A1 & QT00037793 & GATTCCCTGGACCTAAAGGTGC & CTGCTCCATCTGCTTGAAGACC \\
TAGLN & QT00072247 & TCCAGGTCTGGCTGAAGAATGG & \\
Housekeeping gene & & & ACCACCCTGTTGCTGTAGCCAA \\
GAPDH & QT00079247 & GTCTCCTCTGACTTCAACAGCG
\end{tabular}

temperature. Fibroblasts were washed twice in PBS and incubated at $4{ }^{\circ} \mathrm{C}$ overnight with anti-human $\alpha$-SMA (Abcam) diluted at 1:100 in PBS and Triton-X 100 0.05\% solution, anti-human type I collagen (Abcam) diluted at 1: 100 in PBS and Triton-X $1000.05 \%$ solution and with anti-human SM22 (Abcam) diluted at 1:100 in PBS and Triton-X 100 0.05\% solution. Three washes in PBS of the coverslips were followed by $1 \mathrm{~h}$ incubation with goat antimouse IgG-Texas Red-conjugated antibody (Southern Biotechnology, Italy) diluted at 1:100 in PBS and Triton-X 100 0.05\% solution. Cells were then washed twice in PBS and incubated for $10 \mathrm{~min}$ with DAPI solution (diluted 1: 10000) (Abcam). Finally, the coverslips were mounted with Vecta shield (Vector Labs). Fluorescence was examined under an AxioPlan (Zeiss, Oberkochen, Germany) light microscope equipped with epifluorescence at $\times 200$ and $\times 400$ magnification. The negative controls were obtained by omitting the primary antibody. Immunoreactivity of $\alpha$-SMA, Col-1, and SM22 were semi-quantified as the mean densitometric area of $\alpha$-SMA and Col-I signal into the cytoplasm, by AxioVision 4.6 software measure program [25]. At least 100 fibroblasts from each group were evaluated.

\section{Statistical analysis}

Three independent experiments were carried out, and the results were expressed as the mean \pm SD of triplicate values for each experiment. Data normal distribution was evaluated by Shapiro-Wilk, D'Agostino and Pearson, and Kolmogorov-Smirnov tests. Data from realtime PCR were evaluated by one-way ANOVA with a Tukey's post hoc test using $2^{-\Delta \Delta C T}$ values for each sample [26]. All analyses were performed through the SAS System (SAS Institute Inc., Cary, NC, USA) and GraphPad Prism 6.1. A significant value was defined with a $p$ value $<0.05$

\section{Results}

\section{Cell viability and apoptosis}

Results for viability and apoptosis are reported in Fig. 1. At basal levels, the viability of affected LcSSc and DcSSc fibroblasts resulted decreased compared to control ones. LcSSc-unaffected fibroblasts resulted also a bit less viable than control ones, but not at the levels of LcSSc/
DcSSc-affected ones. Anti-Cenp-B and with more extent anti-Topo-I IgGs reduced mainly unaffected LcSSc and control fibroblast (and with less extent affected LcSSc/ DcSSc ones) viability in a dilution-dependent manner compared to control IgGs. Similar results were obtained with anti-Cenp $\mathrm{B}^{+}$and anti-Topo- $\mathrm{I}^{+}$sera compared to control sera and to SSc sera negative for anti-Cenp-B, anti-Topo-I antibodies and for other ENAs. Flow cytometry analysis revealed that both anti-Cenp-B/anti-Topo-I IgGs and anti-Cenp $\mathrm{B}^{+} /$anti-Topo- $\mathrm{I}^{+}$sera induce apoptosis in unaffected LcSSc and control fibroblasts only, while affected LcSSc and DcSSc fibroblasts showed apoptosis resistance. Anti-histone H3 antibody treatment did not influence viability and apoptosis (Additional file 1). Regarding the IAP, AZD 5582 dihydrochloride showed its efficacy in inhibiting apoptosis and increase viability in control fibroblasts upon stimulation with anti-Cenp-B, anti-Topo-I, and antiHistone H3 IgGs (Additional file 1).

\section{Gene expression}

Gene expression levels are reported in Fig. 2. At basal levels, ACTA2, COL1A1, and TAGLN are statistically higher in affected LcSSc and DcSSc fibroblasts compared to control ones. Stimulation with anti-Cenp-B and anti-Topo-I IgGs statistically increased all the profibrotic markers compared to control IgGs. Control and unaffected LcSSc fibroblasts seem to be more prone to IgG stimulation than affected LcSSc/DcSSc ones. Stimulation with anti-Cenp- $\mathrm{B}^{+}$and anti-Topo- $\mathrm{I}^{+}$sera increased ACTA2, COL1A1, and TAGLN expressions compared to control sera and to SSc Cenp- $\mathrm{B}^{-} /$Topo- $^{-}$sera for all the fibroblast populations. Finally, inhibition of apoptosis did not change the mRNA upregulation of all the profibrotic markers upon stimulation with anti-Cenp-B, anti-Topo-I, and anti-Histone H3 IgGs in control fibroblasts (Additional file 1).

\section{Immunofluorescence}

Results for immunofluorescence are shown in Fig. 3. At basal levels (before the treatment), $\alpha$-SMA, Col-I, and SM22 are statistically higher in affected LcSSc and DcSSc fibroblasts compared to control ones. Stimulation with anti-Cenp-B and anti-Topo-I IgGs and with anti- 


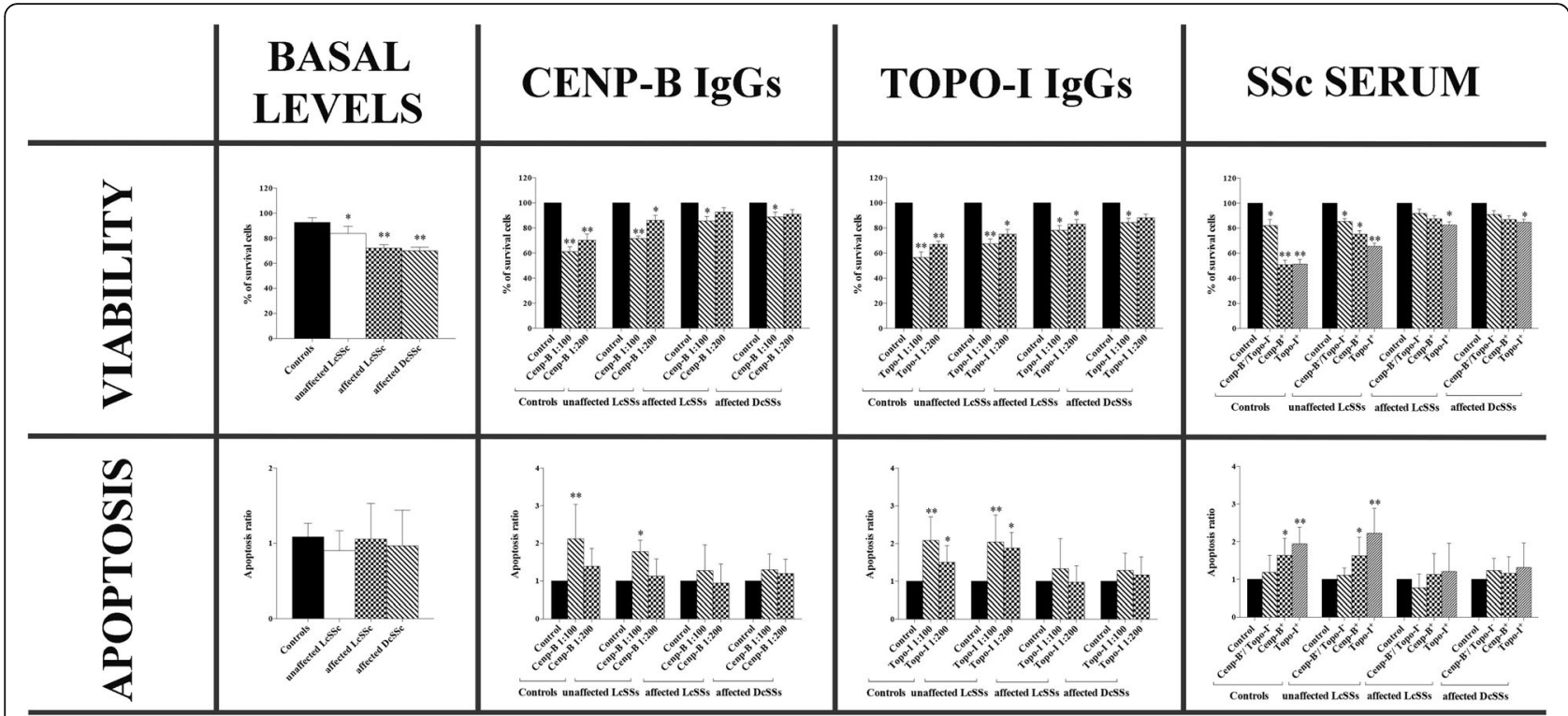

Fig. 1 Viability (top row) and apoptosis (bottom row) detected in control, unaffected LcSSC, affected LcSSC, and affected DcSSc fibroblasts at basal levels (untreated) and after stimulation with anti-Cenp-B (ratios 1:100 and 1:200)/anti-Topo-I (ratios 1:100 and 1:200) antibodies and with SSC sera $\left(10 \% \mathrm{v} / \mathrm{V}\right.$ in DMEM). Data were normalized per $10^{4}$ cells. The statistics is reported with respect to the "Control." "Control" for IgG stimulations is referred to human healthy control IgGs in a ratio 1:100 in culture medium (DMEM). "Control" for serum stimulation is referred to as human healthy control serum at $10 \% \mathrm{~V} / \mathrm{V}$ in DMEM. $\left({ }^{*} p<0.05,{ }^{* *} p<0.01\right)$. For SSc serum treatments, Cenp-B-/Topo-I treatment refers to SSc serum negative for all ENAs

Cenp- $\mathrm{B}^{+}$and anti-Topo- $\mathrm{I}^{+}$sera statistically increased all the profibrotic markers compared to control IgGs, to control sera and to SSc sera negative for anti-Cenp-B and anti-Topo-I antibodies and for other ENAs. In Fig. 4, representative images of ICC assay for all the three markers ( $\alpha$-SMA, Col-I, and SM22) confirm consistency with the ICC quantification data. Same as for qPCR, inhibition of apoptosis did not change the protein upregulation of all the pro-fibrotic markers upon stimulation with anti-Cenp-B, anti-Topo-I, and anti-Histone H3 IgGs in control fibroblasts (Additional file 1).

\section{Discussion}

To the best of our knowledge, this is the first study in which the direct effect of antibodies targeting SScspecific ENAs that are anti-Cenp-B and anti-Topo-I has been evaluated on the pro-fibrotic activation of cultured human dermal fibroblasts and their subsequent differentiation into a myofibroblast phenotype in vitro. SScspecific antibodies have been used mainly as indicators of clinical subsets of the disease [27]. Moreover, these antibodies are as important tools for the prediction of possible organ involvement [28]. However, very little is known about their direct pathogenic effect on different cell phenotypes in the disease [29]. What is known in the population of SSc antibody patients is that patients with anti-Cenp-B antibodies (usually LcSSc patients) more frequently develop pulmonary arterial hypertension $(\mathrm{PAH})$ and prolonged gastrointestinal transit time
[30], while SSc patients with anti-Topo-I antibodies (usually DcSSc patients) are linked with a higher probability of interstitial lung disease (ILD), renal vascular damage, renal crisis, and heart fibrosis [31]. All these internal organ complications involve the fibroblast as the key effector cell phenotype driving the fibrotic process in SSc [32]: therefore, there must be a direct and/or indirect link between the presence of anti-Cenp-B/antiTopo-I antibodies and the pro-fibrotic activation of fibroblasts. In literature, there are some hypotheses on how those antibodies could indirectly mediate the fibrotic development in SSc [33, 34]. Among these, the hypothesis that SSc-specific antibodies could trigger the fibrotic development by inducing microvascular alterations and subsequent tissue remodeling is one of the most reliable [35]. Another important hypothesis is that SSc-specific antibodies form immune complexes (ICs) upon their interaction with soluble target antigens [36]: it has been demonstrated that ICs containing anti-CenpB/anti-Topo-I antibodies induce a pro-fibrotic and proinflammatory phenotype in dermal fibroblasts [37]. Particularly, scientists demonstrated that Topo-I binding to fibroblast surfaces is both necessary and sufficient for anti-Topo-I binding [38]. Second, Topo-I/anti-Topo-I complex binding can then trigger the adhesion and activation of monocytes, thus providing a plausible model for the amplification of the fibrogenic cascade in antiTopo-I-positive SSc patients [39]. To some extent, this model looks very artificial since Topo-I should be an 


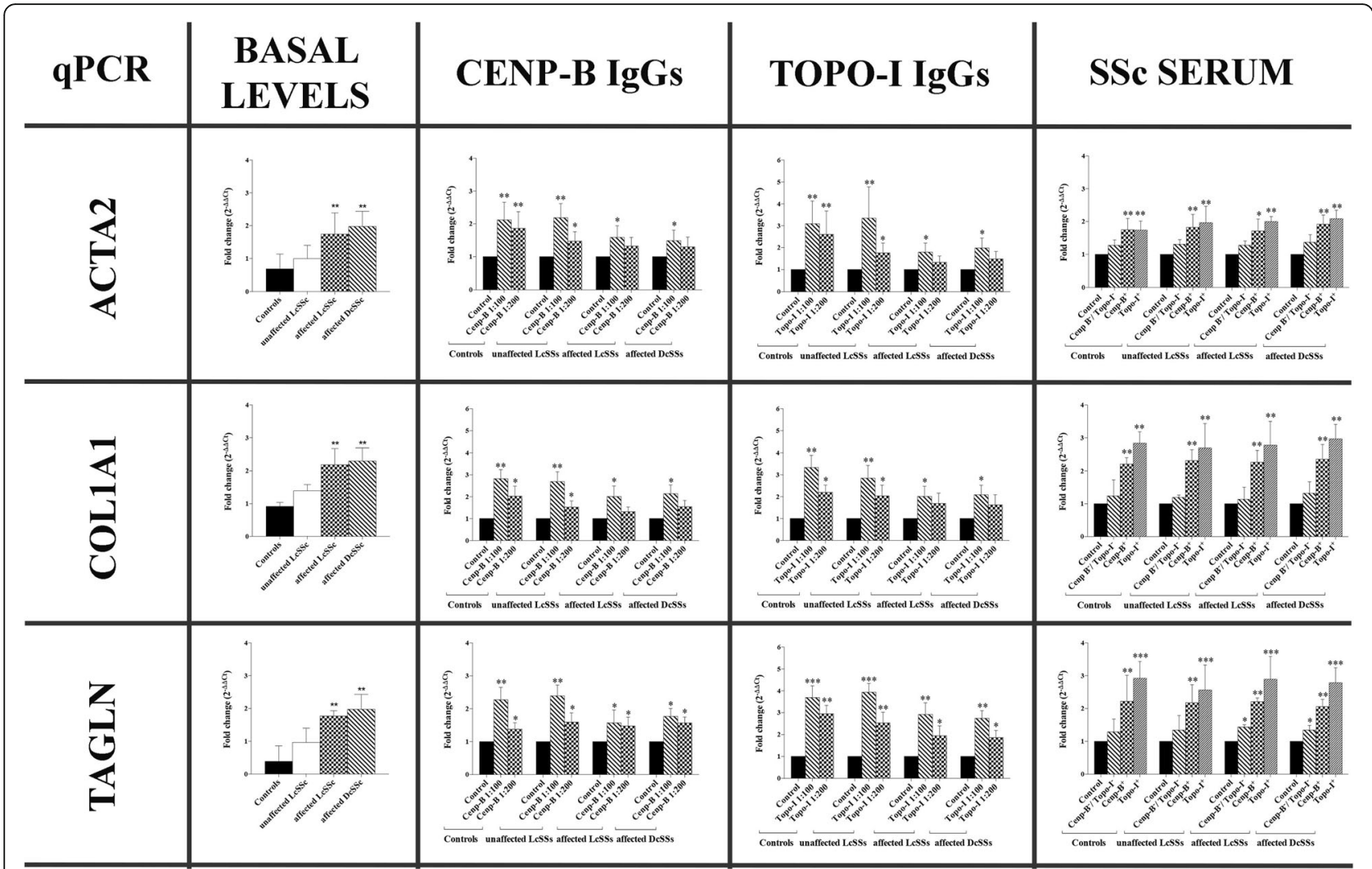

Fig. 2 qPCR results for ACTA2 (top row), COL1A1 (middle row), and TAGLN (bottom row) in control, unaffected LCSSc, affected LCSSC, and affected DcSSc fibroblasts at basal levels (untreated) and after stimulation with anti-Cenp-B (ratios 1:100 and 1:200)/anti-Topo-I (ratios 1:100 and 1:200) antibodies and with SSc sera (10\% v/V in DMEM). The statistics is reported with respect to the "Control." "Control" for IgG stimulations is referred to human healthy control IgGs in a ratio 1:100 in culture medium (DMEM). "Control" for serum stimulation is referred to as human healthy control serum at 10\% v/v in DMEM. Data are reported as fold change vs "Control" $\left.{ }^{*} p<0.05,{ }^{* *} p<0.01,{ }^{* * *} p<0.001\right)$

intracellular antigen (it is usually located in the nucleus) and not an extracellular one [40]. However, subsequent in vivo studies demonstrated that Topo-I released from injured endothelial cells could bind to bystander fibroblasts thus displaying chemoattractant activity toward immature dendritic cells and human monocytes [41]. These results represent a clear demonstration that Topo-I could display an extracellular role that can affect fibroblast physiology. Similar results were found for Cenp-B antigen [42]: in particular, scientists demonstrated that Cenp-B released from apoptotic endothelial cells in vivo binds more specifically to the surface of human pulmonary artery smooth muscle cells (SMCs) than fibroblasts [43]; in particular, Cenp-B binds preferentially to SMCs of the contractile type rather than to SMCs of the synthetic type [44]. The different Cenp-B selectivity of binding to SMCs rather than to fibroblasts could explain our results showing that anti-Topo-I antibodies have a stronger effect on fibroblasts than anti-Cenp-B ones. The different target cells of Cenp-B (SMCs) and Topo-I (fibroblasts) autoantigens released by apoptotic endothelial cells could also partially explain the different complications and organ involvement between LcSSc and DcSSc [45]. Both Cenp-B and Topo-I models assume an already established endothelial damage as a source of autoantigens binding to targeting cells and generating a specific pathogenic autoantibody (antiCenp-B and anti-Topo-I) response. However, these models are quite in disagreement with the timing of the disease evolution since those specific circulating autoantibodies could be detected in SSc patients before an established endothelial damage [46]. This is also the reason why in the past the use of Topo-I inhibitors for the treatment of SSc patients resulted unsuccessful [47]. With the results of the present study, we assume that anti-Cenp-B and anti-Topo-I antibodies could exert a direct pathogenic role, and therefore, they could be considered "functional antibodies." In fact, an autoantibody is considered "functional" if its direct interaction with an identified target antigen leads to a molecular pathway activation or inhibition that can be replicated in an experimental setting [48]. Therefore, the results of the present study suggest a new interpretation of the role of anti-Cenp-B and anti-Topo-I antibodies in the disease in 

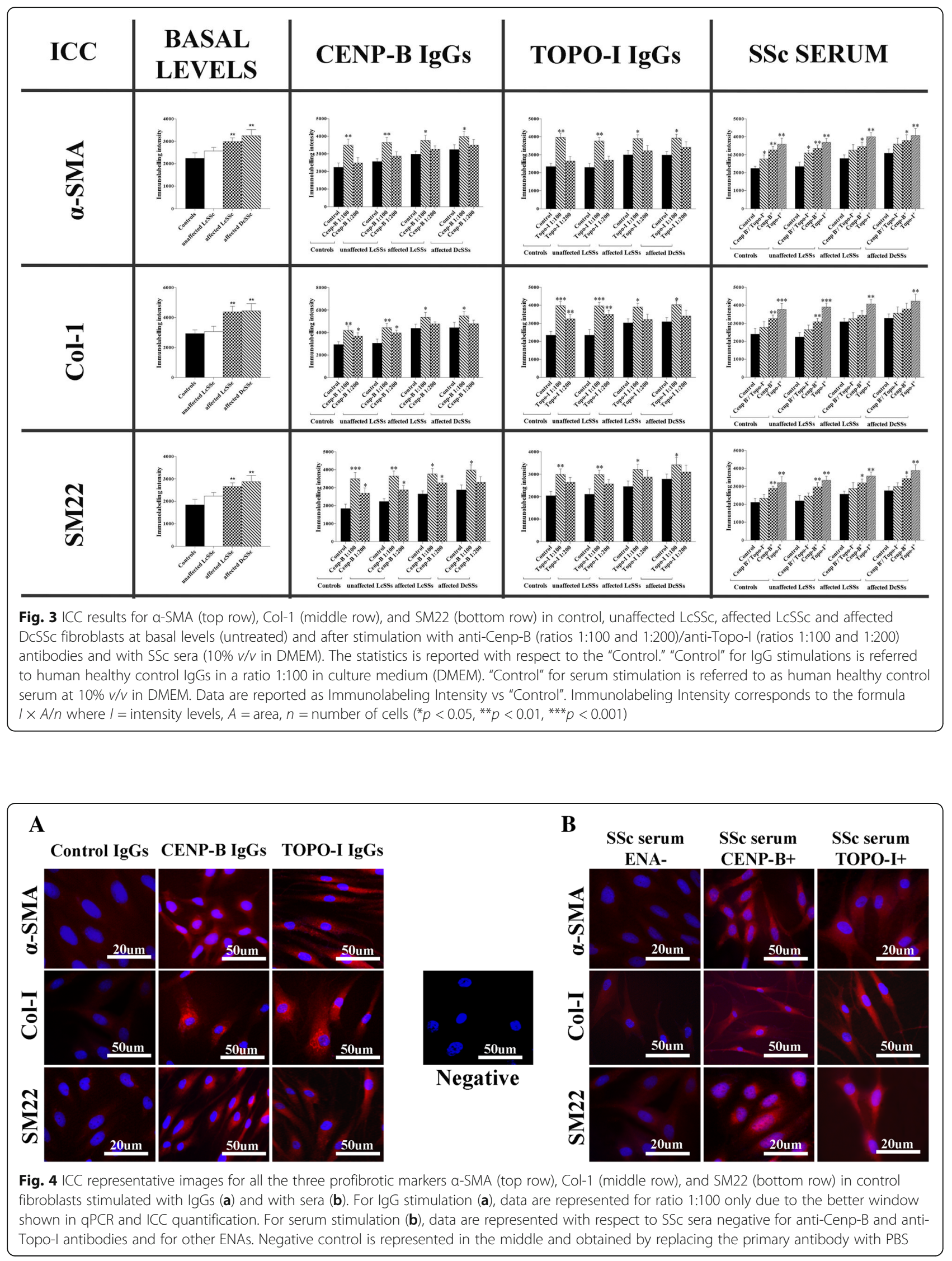
terms of disease drivers and not only representing an epiphenomenon and/or useful diagnostic and prognostic tools. In fact, we believe that these main autoantibodies, when present in the circulation even years before the clinical involvement, need to be tackled to slow down or prevent disease development. To prove this hypothesis, we have support from literature: it has been demonstrated that ubiquitous nuclear protein Cenp- $B$ is the main target of anti-endothelial cell antibodies (AECA) in patients with LcSSc and that AECA from DcSSc patients bind to endothelial cell topoisomerase I, suggesting that classical autoantibodies such as anti-Cenp-B and antiTopo-I antibodies could act as AECA inducing cellmediated toxicity and apoptosis in the early stages of the disease [49]. In this scenario, the use of immunosuppressive drugs and/or the development of more specific drugs targeting anti-Cenp-B and anti-Topo-I antibodies should be recommended at an early stage of the disease to prevent future organ damage or decrease the fibrotic evolution. In this regard, we underline that in the literature, to our knowledge, there are no studies on the progress and the possible decrease of the autoantibody titer during immunosuppressive therapy. Another important finding evidenced in this work is the different responsiveness of the fibroblasts to those autoantibodies according to their differentiation stage: healthy and unaffected LcSSc fibroblasts were more prone to be activated upon stimulation with anti-Cenp-B and antiTopo-I antibodies than affected LcSSc and DcSSc fibroblasts that resulted already differentiated into activated myofibroblasts, thus secreting the maximum level of pro-fibrotic proteins. On the other hand, healthy and unaffected LcSSc fibroblasts were also more susceptible to apoptosis than affected LcSSc and DcSSc ones, suggesting that the fate of fibroblasts depends not only on autoantibodies but on a combination of specific autoantibodies (e.g., anti-histone antibodies did not induce apoptosis) and other soluble factors: in fact what we call healthy or unaffected LcSSc fibroblasts are cells that, upon stimulation with autoantibodies, they secrete a maximum amount of pro-fibrotic proteins and then undergo apoptosis. On the other hand, what we call affected LcSSc and DcSSc fibroblasts are cells that already express high amount of pro-fibrotic proteins and, upon stimulation with autoantibodies, they keep producing collagen and other contractile proteins but with apoptosis resistance, so the self-sustained pro-fibrotic loop is established [50]. This theory is in line with recently published literature in which it has been demonstrated that mitochondria in activated myofibroblasts, but not quiescent fibroblasts, are primed by death signals (proximity to the apoptotic threshold) which creates a requirement for tonic expression of the antiapoptotic proteins to ensure myofibroblast survival [51]. In this irreversible loop, the inefficient removal of nuclear components of cells targeted by the autoantibodies (defective cellular "waste disposal" theory) may also lead to the release and prolonged exposure of nuclear components and thus to the generation of new autoantibodies with increased or stable titers in sera of SSc patients.

\section{Conclusions}

In conclusion, we suggest to re-consider the use of SScspecific antibodies not only as useful diagnostic and prognostic tools, but also as therapeutic targets of the disease itself. We acknowledge that our study presents intrinsic limitations. Being an in vitro study, it might be oversimplified, not allowing an adequate reproduction of the complexity of the disease pathogenesis. Moreover, the direct pathogenic role of other SSc-related autoantibodies (e.g., anti-RNA polymerase III) needs to be further investigated.

\section{Additional file}

Additional file 1: Viability and apoptosis (left panel) and qPCR and ICC (right panel) data regarding control fibroblasts stimulated with specific SSC-autoantibodies (anti-Cenp-B, anti-Topo-I IgGs 1:100) and with SScunrelated one (anti-Histone $\mathrm{H} 3 \mathrm{lgGs}$ 1:100) with and without the preincubation ( $2 \mathrm{~h}$ ) with an anti-apoptotic compound (IAP, AZD 5582 dihydrochloride, $50 \mathrm{nM})\left({ }^{*} p<0.05\right)$. (TIF $\left.42160 \mathrm{~kb}\right)$

\section{Abbreviations}

ACA: Anti-centromere antibody; ACR: American College of Rheumatology; AECA: Anti-endothelial cell antibodies; ANA: Anti-nuclear antibody; AntiCenp-B: Anti-centromeric protein B; Anti-elF2B: Anti-eukaryotic initiation factor 2B; Anti-RNAP: Anti-RNA polymerase III; Anti-RNP: Anti-U3 ribonucleoprotein; Anti-Topo-l: Anti-DNA-topoisomerase-l; Col-I: Type I collagen; DcSSc: Diffuse cutaneous systemic sclerosis; DMEM: Dulbecco's modified Eagle's medium; DMSO: Dimethyl sulfoxide; ENA: Extractable nuclear antigen; EULAR: European league against rheumatism; FBS: Fetal bovine serum; FITC: Fluorescein isothiocyanate; GAPDH: Glyceraldehyde 3phosphate dehydrogenase; IC: Immune complex; ICC: Immunocytochemistry; ILD: Interstitial lung disease; LCSSc: Limited cutaneous systemic sclerosis; MTT: 3-(4,5-Dimethylthiazol-2-yl)-2,5-diphenyltetrazolium bromide; OD: Optical density; PAH: Pulmonary arterial hypertension; PBS: Phosphatebuffered saline; PI: Propidium iodide; qPCR: Quantitative real-time polymerase chain reaction; SD: Standard deviation; SM22: Trangelin; SMC: Smooth muscle cell; SSc: Systemic sclerosis; a-SMA: Alpha smooth muscle actin

\section{Acknowledgements \\ Not applicable.}

\section{Authors' contributions}

CC conceived of and designed the study interpreted the data, wrote and revised the manuscript. SC performed the fibroblast isolation from skin biopsies and the cell cultures and treatments, interpreted the data, and revised the manuscript. AF contributed to the enrolment of patients with SSC and harvested information about clinical and demographic parameters. DF performed GPCR experiments and interpreted the data. NV performed ICC staining, performed the statistical analysis and interpreted the data, created the figures, and wrote and revised the manuscript. MC, SS, and RN revised the manuscript for critical and important intellectual content. NG conceived of and designed the study, performed the skin biopsies, collected and interpreted the data, performed the literature search, and wrote and revised the manuscript. All authors read and approved the final manuscript, and all authors agree to be accountable for all aspects of the study. 


\section{Funding}

Not applicable (nothing to declare).

\section{Availability of data and materials}

Please contact the author for data requests.

\section{Ethics approval and consent to participate}

All patients gave their fully informed, voluntary, and written consent according to the principles of the Declaration of Helsinki and in compliance with the ethics committee of the University of Siena, whose institutional review board approved the entire study protocol.

\section{Consent for publication}

Not applicable.

\section{Competing interests}

The authors declare that they have no competing interests.

\section{Author details}

'Scleroderma Unit, Department of Medicine, Surgery and Neurosciences, University of Siena, Siena, Italy. ${ }^{2}$ Rheumatology Unit, Department of Medicine, Surgery and Neurosciences, University of Siena, Siena, Italy. ${ }^{3}$ Research Laboratory and Academic Division of Clinical Rheumatology, Department of Internal Medicine, University of Genoa, Genoa, Italy.

\section{Received: 18 March 2019 Accepted: 5 June 2019}

\section{Published online: 24 June 2019}

\section{References}

1. Denton CP. Advances in pathogenesis and treatment of systemic sclerosis Clin Med (Lond). 2016;16:55-60.

2. Rajkumar VS, Howell K, Csiszar K, Denton CP, Black CM, Abraham DJ. Shared expression of phenotypic markers in systemic sclerosis indicates a convergence of pericytes and fibroblasts to a myofibroblast lineage in fibrosis. Arthritis Res Ther. 2005;7:1113-23.

3. LeRoy EC, Black C, Fleischmajer R, Jablonska S, Krieg T, Medsger TA Jr, et al, Scleroderma (systemic sclerosis): classification, subsets and pathogenesis. J Rheumatol. 1988;15:202-5.

4. Allanore Y. Limited cutaneous systemic sclerosis: the unfairly neglected subset. J Scleroderma Relat Disord. 2016:1:241-6.

5. Pearson DR, Werth VP, Pappas-Taffer L. Systemic sclerosis: current concept of skin and systemic manifestations. Clin Dermatol. 2018:36:459-74.

6. Stochmal, A., Czuwara, J., Trojanowska, M. et al. Clinic Rev Allerg Immunol. 2019; https://doi.org/10.1007/s12016-018-8718-8

7. Banhuk FW, Pahim BC, Jorge AS, Menolli RA. Relationships among antibodies against extractable nuclear antigens, antinuclear antibodies, and autoimmune diseases in a Brazilian public hospital. Autoimmune Dis. 2018, 2018:9856910

8. Okano Y. Antinuclear antibody in systemic sclerosis (scleroderma). Rheum Dis Clin N Am. 1996;22:709-35.

9. Tan EM. Antinuclear antibodies: diagnostic markers for autoimmune diseases and probes for cell biology. Adv Immunol. 1989:44:93-151.

10. Tartar DM, Chung L, Fiorentino DF. Clinical significance of autoantibodies in dermatomyositis and systemic sclerosis. Clin Dermatol. 2018;36:508-24.

11. Choi MY, Fritzler MJ. Progress in understanding the diagnostic and pathogenic role of autoantibodies associated with systemic sclerosis. Curr Opin Rheumatol. 2016;28:586-94.

12. Liaskos C, Marou E, Simopoulou T, Barmakoudi M, Efthymiou G, Scheper T, et al. Disease-related autoantibody profile in patients with systemic sclerosis. Autoimmunity. 2017;50:414-21.

13. Kuwana M. Circulating anti-nuclear antibodies in systemic sclerosis: utility in diagnosis and disease subsetting. J Nippon Med Sch. 2017;84:56-63.

14. Boonstra M, Mertens BJA, Bakker JA, Ninaber MK, Ajmone Marsan N, van der Helm-van Mil AHM, et al. To what extent do autoantibodies help to identify high-risk patients in systemic sclerosis? Clin Exp Rheumatol. 2018;36 Suppl 113(4):109-17

15. Van den Hoogen F, Khanna D, Fransen J, Johnson SR, Baron M, Tyndall A, et al. 2013 classification criteria for systemic sclerosis: an American College of Rheumatology/European league against rheumatism collaborative initiative. Ann Rheum Dis. 2013;72:1747-55.
16. Van den Hoogen F, Khanna D, Fransen J, Johnson SR, Baron M, Tyndall A, et al. 2013 classification criteria for systemic sclerosis: an American College of Rheumatology/European league against rheumatism collaborative initiative. Arthritis Rheum. 2013;65:2737-47.

17. Valentini G, Marcoccia A, Cuomo G, Vettori S, ludici M, Bondanini F, et al. Early systemic sclerosis: analysis of the disease course in patients with marker autoantibody and/or capillaroscopic positivity. Arthritis Care Res. 2014;66:1520-7.

18. Salazar GA, Assassi S, Wigley F, Hummers L, Varga J, Hinchcliff M, et al. Antinuclear antibody-negative systemic sclerosis. Semin Arthritis Rheum. 2015;44:680-6

19. Chander R, Singh S, Kalantri SA, Charan S, Gupta A. Sero-negative systemic sclerosis: a rare presentation. J Clin Diagn Res. 2016:10:OD12-3.

20. Clements PJ, Lachenruch PA, Nq SC, Simmons M, Sterz M, Furst DE. Skin score. A semiquantitative measure of cutaneous involvement that improves prediction of prognosis in systemic sclerosis. Arthritis Rheum. 1990:33:1256-63.

21. Cheleschi S, Giordano N, Volpi N, Tenti S, Gallo I, Di Meglio M, et al. A complex relationship between Visfatin and Resistin and microRNA: an in vitro study on human chondrocyte cultures. Int J Mol Sci. 2018;19:3909.

22. Ramakers C, Ruijter JM, Deprez RH, Moorman AF. Assumption-free analysis of quantitative real-time polymerase chain reaction (PCR) data. Neurosci Lett. 2003;339:62-6.

23. Pfaffl MW. A new mathematical model for relative quantification in real RTPCR. Nucleic Acid Res. 2001;29:e45.

24. Vandesompele J, de Preter K, Pattyn F, Poppe B, van Roy N, de Paepe A, et al. Accurate normalization of real-time quantitative RT-PCR data by geometric averaging of multiple internal control genes. Genome Biol. 2002; 3:research0034.1.

25. Corallo C, Franci B, Lucani B, Montella A, Chirico C, Gonnelli S, et al. From microvasculature to fibroblasts: contribution of anti-endothelial cell antibodies in systemic sclerosis. Int J Immunopathol Pharmacol. 2015;28:93103.

26. Corallo C, Santucci A, Bernardini G, Figura N, Leoncini R, Riolo G, et al. Proteomic investigation of dermal fibroblasts isolated from affected and unaffected skin samples from patients with limited cutaneous systemic sclerosis: 2 distinct entities? J Rheumatol. 2017:44:40-8.

27. Mehra S, Walker J, Patterson K, Fritzler MJ. Autoantibodies in systemic sclerosis. Autoimmun Rev. 2013;12:340-54.

28. Kranenburg $P$, van den Hombergh WM, Knaapen-Hans HK, van den Hoogen $\mathrm{FH}$, Fransen J, Vonk MC. Survival and organ involvement in patients with limited cutaneous systemic sclerosis and anti-topoisomerase-I antibodies: determined by skin subtype or auto-antibody subtype? A long-term followup study. Rheumatology (Oxford). 2016;55:2001-8.

29. Steen VD. Autoantibodies in systemic sclerosis. Semin Arthritis Rheum. 2005; 35:35-42.

30. Coghlan JG, Denton CP, Grunig E, Bonderman D, Distler O, Khanna D, et al. Evidence-based detection of pulmonary arterial hypertension in systemic sclerosis: the DETECT study. Ann Rheum Dis. 2014;73:1340-9.

31. Hesselstrand R, Scheja A, Shen GQ, Wiik A, Akesson A. The association of antinuclear antibodies with organ involvement and survival in systemic sclerosis. Rheumatology (Oxford). 2003;42:534-40.

32. van Caam $A$, Vonk $M$, van den Hoogen $F$, van Lent $P$, van der Kraan $P$. Unraveling SSc pathophysiology; the Myofibroblast. Front Immunol. 2018;9:2452.

33. Nunes JPL, Cunha AC, Meirinhos T, Nunes A, Araújo PM, Godinho AR, et al. Prevalence of auto-antibodies associated to pulmonary arterial hypertension in scleroderma - a review. Autoimmun Rev. 2018;17:1186-201.

34. Kim D, Peck A, Santer D, Patole P, Schwartz SM, Molitor JA, et al. Induction of interferon-alpha by scleroderma sera containing autoantibodies to topoisomerase I: association of higher interferon-alpha activity with lung fibrosis. Arthritis Rheum. 2008:58:2163-73.

35. Schulz JN, Plomann M, Sengle G, Gullberg D, Krieg T, Eckes B. New developments on skin fibrosis - essential signals emanating from the extracellular matrix for the control of myofibroblasts. Matrix Biol. 2018;69:522-32.

36. Ohyama K, Baba M, Tamai M, Aibara N, Ichinose K, Kishikawa N, et al. Proteomic profiling of antigens in circulating immune complexes associated with each of seven autoimmune diseases. Clin Biochem. 2015;48:181-5.

37. Raschi E, Chighizola CB, Cesana L, Privitera D, Ingegnoli F, Mastaglio C, et al. Immune complexes containing scleroderma-specific autoantibodies induce a profibrotic and proinflammatory phenotype in skin fibroblasts. Arthritis Res Ther. 2018;20:187. 
38. Hénault J, Robitaille G, Senécal JL, Raymond Y. DNA topoisomerase I binding to fibroblasts induces monocyte adhesion and activation in the presence of anti-topoisomerase I autoantibodies from systemic sclerosis patients. Arthritis Rheum. 2006:54:963-73.

39. Hénault J, Tremblay M, Clément I, Raymond Y, Senécal JL. Direct binding of anti-DNA topoisomerase I autoantibodies to the cell surface of fibroblasts in patients with systemic sclerosis. Arthritis Rheum. 2004;50:3265-74.

40. Girstun A, Ishikawa T, Kowalska-Loth B, Czubaty A, Staron K. Subnuclear localization of human topoisomerase I. J Cell Biochem. 2017;1 18:407-19.

41. Oppenheim JJ, Dong HF, Plotz P, Caspi RR, Dykstra M, Pierce S, et al. Autoantigens act as tissue-specific chemoattractants. J Leukoc Biol. 2005;77: 854-61.

42. Robitaille G, Henault J, Christin MS, Senecal JL, Raymond Y. The nuclear autoantigen CENP-B displays cytokine-like activities toward vascular smooth muscle cells. Arthritis Rheum. 2007:56:3814-26.

43. Robitaille G, Christin MS, Clement I, Senecal JL, Raymond Y. Nuclear autoantigen CENP-B transactivation of the epidermal growth factor receptor via chemokine receptor 3 in vascular smooth muscle cells. Arthritis Rheum. 2009;60:2805-16.

44. Howard OZ. Autoantigen signalling through chemokine receptors. Curr Opin Rheumatol. 2006;18:642-6.

45. Ingegnoli F, Ughi N, Mihai C. Update on the epidemiology, risk factors, and disease outcomes of systemic sclerosis. Best Pract Res Clin Rheumatol. 2018; 32:223-40.

46. Orlandi M, Barsotti S, Lepri G, Codullo V, Di Battista M, Guiducci S, et al. One year in review 2018: systemic sclerosis. Clin Exp Rheumatol. 2018;36(Suppl 113):3-23.

47. Rudnicka L, Czuwara J, Barusińska A, Nowicka U, Makieła B, Jabłonska S. Implications for the use of topoisomerase I inhibitors in treatment of patients with systemic sclerosis. Ann N Y Acad Sci. 1996;803:318-20.

48. Günther J, Rademacher J, van Laar JM, Siegert E, Riemekasten G. Functional autoantibodies in systemic sclerosis. Semin Immunopathol. 2015;37:529-42.

49. Mihai C, Tervaert JW. Anti-endothelial cell antibodies in systemic sclerosis. Ann Rheum Dis. 2010;69(2):319-24.

50. Vahidi Manesh P, Farazmand A, Gharibdoost F, Vanaki N, Mostafaei S, Kavosi $\mathrm{H}$, et al. Downregulation of miR-542-3p contributes to apoptosis resistance in dermal fibroblasts from systemic sclerosis patients via Survivin overexpression. Iran J Allergy Asthma Immunol. 2019;18(2):173-81.

51. Lagares D, Santos A, Grasberger PE, Liu F, Probst CK, Rahimi RA, et al. Targeted apoptosis of myofibroblasts with the BH3 mimetic ABT-263 reverses established fibrosis. Sci Transl Med. 2017;9(420):eaal3765.

\section{Publisher's Note}

Springer Nature remains neutral with regard to jurisdictional claims in published maps and institutional affiliations.

Ready to submit your research? Choose BMC and benefit from:

- fast, convenient online submission

- thorough peer review by experienced researchers in your field

- rapid publication on acceptance

- support for research data, including large and complex data types

- gold Open Access which fosters wider collaboration and increased citations

- maximum visibility for your research: over $100 \mathrm{M}$ website views per year

At $\mathrm{BMC}$, research is always in progress.

Learn more biomedcentral.com/submissions 